\title{
BMJ Open Impact evaluation of the Care Tipping Point Initiative in Nepal: study protocol for a mixed-methods cluster randomised controlled trial
}

Kathryn M Yount (1) , ${ }^{1}$ Cari Jo Clark, ${ }^{2}$ Irina Bergenfeld, ${ }^{2}$ Zara Khan, ${ }^{2}$ Yuk Fai Cheong, ${ }^{3}$ Sadhvi Kalra, ${ }^{4}$ Sudhindra Sharma, ${ }^{5}$ Shuvechha Ghimire, ${ }^{5}$ Ruchira T Naved, ${ }^{6}$ Kausar Parvin, ${ }^{6}$ Mahfuz Al Mamun, ${ }^{6}$ Aloka Talukder, ${ }^{6}$ Anne Laterra, ${ }^{7}$ Anne Sprinkel, ${ }^{4}$ on behalf of the Tipping Point Program Study Team

To cite: Yount KM, Clark CJ, Bergenfeld I, et al. Impact evaluation of the Care Tipping Point Initiative in Nepal: study protocol for a mixedmethods cluster randomised controlled trial. BMJ Open 2021;11:e042032. doi:10.1136/ bmjopen-2020-042032

- Prepublication history and additional online supplemental material for this paper are available online. To view these files, please visit the journal online (http://dx.doi.org/10. 1136/bmjopen-2020-042032)

Received 24 June 2020 Accepted 13 July 2021

Check for updates

(C) Author(s) (or their employer(s)) 2021. Re-use permitted under CC BY-NC. No commercial re-use. See rights and permissions. Published by BMJ.

For numbered affiliations see end of article.

Correspondence to Dr Kathryn M Yount; kyount@emory.edu

\section{ABSTRACT}

Introduction Girl child, early and forced marriage (CEFM) persists in South Asia, with long-term consequences for girls. CARE's Tipping Point Initiative (TPI) addresses the causes of CEFM by challenging repressive gender norms and inequalities. The TPI engages different participant groups on programmatic topics and supports community dialogue to build girls' agency, shift inequitable power relations, and change community norms sustaining CEFM.

Methods/analysis The Nepal TPI impact evaluation has an integrated, mixed-methods design. The quantitative evaluation is a three-arm, cluster randomised controlled trial (control; Tipping Point Programme (TPP); TPP+ with emphasised social norms change). Fifty-four clusters of $\sim 200$ households were selected from two districts (27:27) with probability proportional to size and randomised. A household census ascertained eligible study participants, including unmarried girls and boys 12-16 years (1242:1242) and women and men 25+ years (270:270). Baseline participation was 1134 girls, 1154 boys, 270 women and 270 men. Questionnaires covered agency; social networks/norms; and discrimination/violence. Thirty in-depth interviews, 8 key-informant interviews and 32 focus group discussions were held across eight TPP/ TPP+ clusters. Guides covered gender roles/aspirations; marriage decisions; girls' safety/mobility; collective action; perceived shifts in child marriage; and norms about girls. Monitoring involves qualitative interviews, focus groups and session/event observations over two visits. Qualitative analyses follow a modified grounded theory approach. Quantitative analyses apply intention to treat, regression-based difference-in-difference strategies to assess impacts on primary (married, marriage hazard) and secondary outcomes, targeted endline tracing and regression-based methods to address potential selection bias.

Ethics/dissemination The Nepal Social Welfare Council approved CARE Nepal to operate in the study districts. Emory (IRB00109419) and the Nepal Health Research Council (161-2019) approved the study. We follow UNICEF and CARE guidelines for ethical research involving children and gender-based violence. Study materials are here
Strengths and limitations of this study

- The Tipping Point Initiative addresses the causes of child, early and forced marriage (CEFM).

- The Nepal Tipping Point Impact Evaluation has an integrated, mixed-methods design.

- The qualitative longitudinal study provides insights about the normative context of CEFM.

- The three-arm cluster-randomised controlled trial assesses incremental effects of norm-change programming.

- Given challenges with programme recruitment, demographics will be compared with local census data and inferences made to the study sample.

or available on request. We will share findings through clinicaltrials.gov, CARE reports/briefs and publications. Trial registration number NCT04015856.

\section{INTRODUCTION}

Child, early and forced marriage: prevalence and causes

Each year, child, early and forced marriage (CEFM), usually defined as marriage before age 18 years, affects more than 10 million girls globally. ${ }^{1}$ These marriages violate girls' rights to bodily autonomy, health, education and opportunity. CEFM also results in lifelong consequences for the physical, emotional, material and psychological well-being of girls. $^{2-4}$ About half of all child marriages occur in South Asia. ${ }^{56}$

Formative research by CARE's Tipping Point Initiative (TPI), a programme aimed at addressing the causes of CEFM in Nepal and Bangladesh, has identified CEFM as a symptom of gender inequality rooted in the control of girls' sexuality and enforced through the exclusion of girls' voices in marriage processes. ${ }^{7}$ In Nepal, child marriage 
is concentrated in specific castes-including Dalits, Madhesi, low-caste Hindus and other economically marginalised castes. ${ }^{67}$ The social and economic isolation of these groups limits their ability to change practices like child marriage, even when aggregate patterns for all castes suggest change. ${ }^{7}$

\section{Interventions to prevent CEFM}

In addition to poor knowledge about causes of CEFM in South Asia, evidence of the impacts of programmes seeking to address CEFM is lacking. In a systematic review of reviews of intervention studies aiming to reduce genderbased violence against adolescent girls, ${ }^{8} 12$ intervention studies cited in high-quality reviews focused on preventing CEFM. Of these, six studies were undertaken in South Asia, and one (quasi-experimental) study was undertaken in Nepal. While a majority of the studies had multiplecomponent, multilevel intervention designs, almost all of those that involved community-engagement/normschange activities with individual-level empowerment activities were small and quasi-experimental, limiting the evidence on these types of interventions. An unpublished review of child-marriage intervention studies not included in the above review of reviews revealed similar findings. ${ }^{9}$

\section{CARE'S TPI}

CARE's TPI focuses on addressing the causes of CEFM and on promoting the rights of adolescent girls through community-level programming focusing on a synchronised engagement of different participant groups to challenge social expectations and repressive gender norms and promote girl-centric and girl-led activism. TPI has designed a 'core' programme package, the Tipping Point Programme (TPP), which includes components, implemented over 18 months, to enhance adolescent girls' personal assets/intrinsic agency (including selfefficacy) and instrumental agency (including voice and negotiation skills). TPI also is testing an enhanced programme package, $\mathrm{TPP}+$, also implemented over 18 months, which includes all elements in TPP plus activities to enhance social norms change by engaging community leaders and by facilitating girl-led community activities. Figure 1 summarises the components and participant groups of the CARE 'core' and 'enhanced' programme models, detailed elsewhere (https://caretippingpoint.org/implementation-manuals/). Programme updates related to COVID-19 are being recorded in the trial registry.

In sum, programmatic gaps to address the causes of CEFM are large, and few, rigorous impact evaluations of social norms and empowerment-based prevention programmes exist. The CARE TPI leveraged its 'core' and 'enhanced' programme models, a cluster randomised controlled trial (C-RCT), and large sample sizes to assess impacts on CEFM and mediating outcomes aligned with the TPP and TPP+ models.

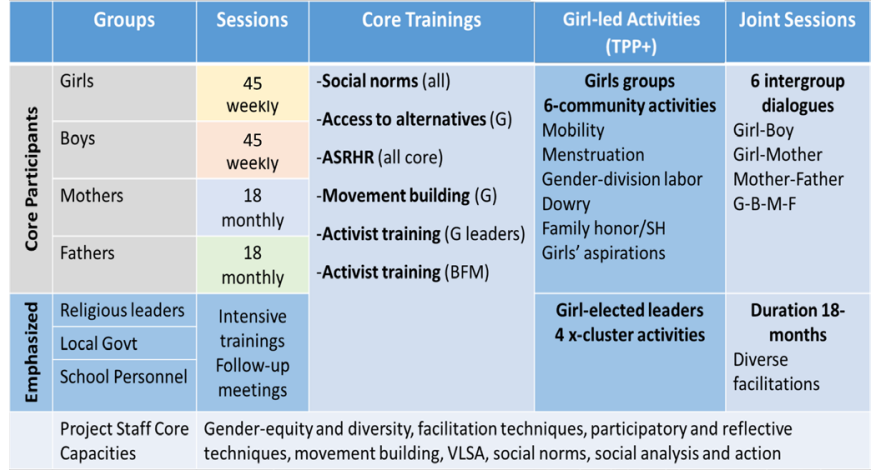

Figure 1 Care Tipping Point Programme (TPP) and Tipping Point Programme plus intervention packages. ASRHR, Adolescent sexual and reproductive health and rights; G, Girl; BFM, Boy-Father-Mother; SH, Sexual Health; VSLA, Village Savings and Loan Association.

\section{METHODS AND ANALYSIS}

\section{Aims and research questions}

This study aims to triangulate data from qualitative and quantitative approaches to evaluate the impacts of the TPP and TPP+ models on CEFM by enhancing adolescent girls' intrinsic, instrumental and collective agency as well as through community social norms diffusion. The following research questions, aligned with the TPI learning components, organise the research. First, to what extent, as a result of the programme(s), do adolescent girls experience decreases in their (a) risk (qualitative) and (b) hazard (quantitative) of first marriage and first gauna.

Second, to what extent, due to the programme(s), do any observed changes in the above primary outcomes operate through increases in the following secondary outcomes related to girls':

- Personal assets and intrinsic agency, including: (1) critical awareness about gender and rights; (2) aspirations and choices regarding education, freedom of movement and marriage; (3) knowledge about SRHR and (4) attitudes about SRHR.

- Instrumental agency, including (1) communication and negotiation skills; (2) capacity to make decisions, (3) practice of SRHR and (4) leadership competence.

- Collective agency or girl-centred movement building, including: (1) group cohesion, solidarity and mobilisation skills and (2) autonomous engagement with different networks, the community and governmental and non-governmental stakeholders to change social norms and claim their rights.

Finally, to what extent, due to the programme(s), do any observed changes in the above primary outcomes operate through shifts in the following secondary outcomes related to the community's:

- Social norms (qualitative and quantitative) with respect to (1) perceptions of what others do in terms of gender, rights (including ASRHR) and CEFM (including dowry); as well as (2) perceptions of what 


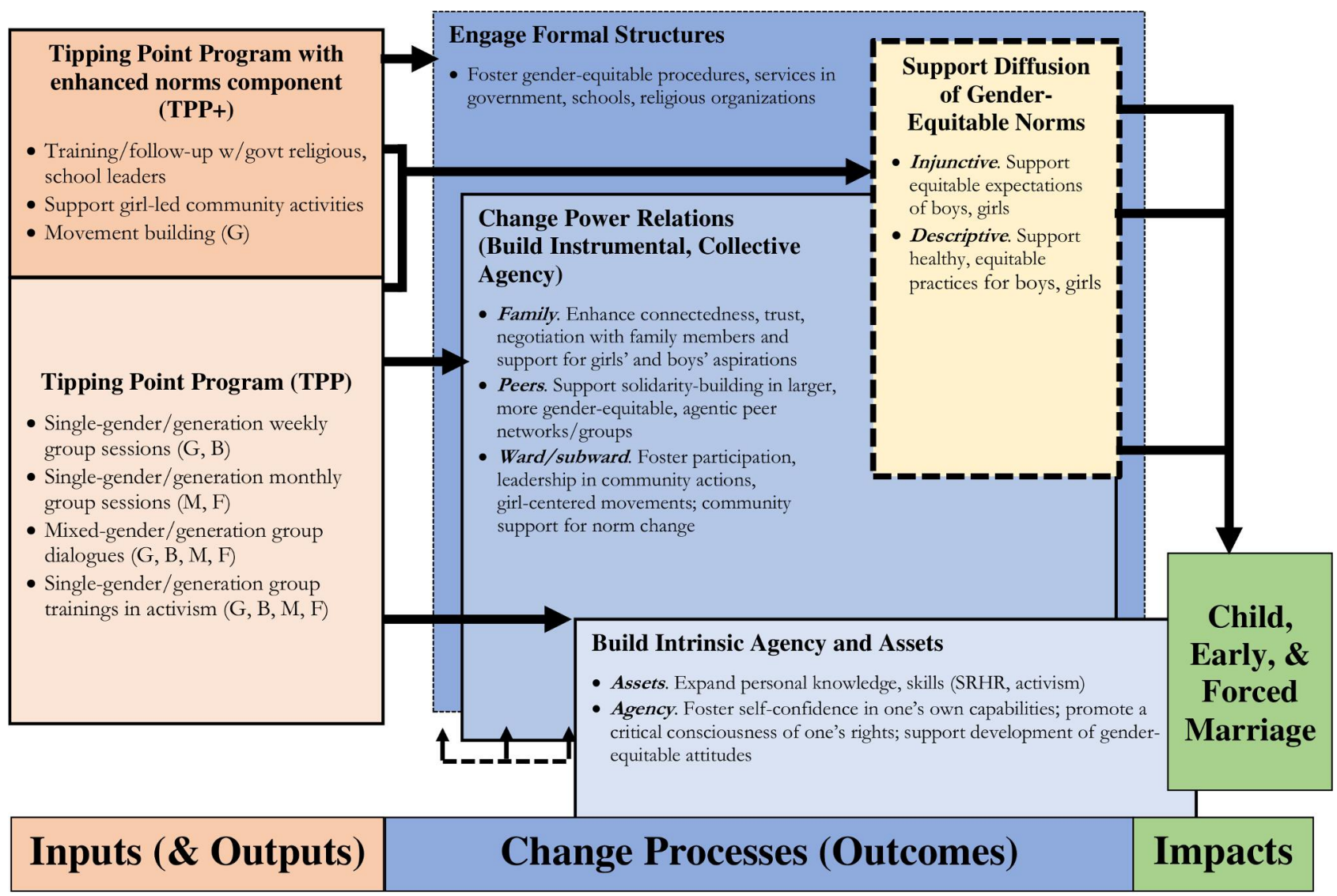

Figure 2 Care Tipping Point Programme theory of change. Dashed arrows denote feedback loops between individual (intrinsic) agency, instrumental and collective agency in various reference groups or social networks, and formal structures in the community. B, boy; F, father; G, girl; M, mother; SRHR, sexual and reproductive health and rights.

others expect them to do in terms of gender, rights (including ASRHR) and CEFM (including dowry).

\section{Setting}

The study sites are Kapilvastu and Rupandehi districts, where the Nepali Government has prioritised CARE programming and where no concurrent CARE or other NGO programming related to CEFM have been underway. The districts have the lowest median ages at first marriage in Nepal (See the website: girlsnotbrides. org https://www.girlsnotbrides.org/child-marriage/ nepal/). Both districts exhibit low levels of human development. Although life expectancies at birth in Rupandehi are above the national average (68.0 vs 66.6 years), life expectancy in Kapilvastu (61.3 years) falls below the national average. Rupandehi and Kapilvastu lag behind the national averages for per capita income (US\$1123 PPP and US $\$ 990$ vs US $\$ 1160$ PPP).

\section{Mixed-methods cluster randomised design}

This study protocol is adapted from a C-RCT developed by the International Centre for Diarrhoeal Disease Research, Bangladesh (icddr,b), to evaluate TPI in Bangladesh and Nepal. The adapted protocol added sampling procedures and study forms from the Room-to-Read Girls Education Programme (RTR-GEP) impact evaluation, ${ }^{10}$ modified study forms developed by icddr,b, added the impact-evaluation theory of change (figure 2) and added analysis plans. The adapted study design entails a mixedmethods, three-arm C-RCT. Arm 1 is the control group. Arm 2 is the TPP group. Arm 3 is the TPP+ group. This design allows for measurement of the effects of:

- TPP on primary and secondary outcomes versus the control condition.

- TPP+ on primary and secondary outcomes vs the control condition.

- TPP+ versus TPP, to assess the incremental effects of emphasised social norms change.

The qualitative evaluation involves data collection with adolescent girls, adolescent boys, parents of adolescents and community stakeholders in eight TPP/TPP+clusters through in-depth interviews (IDIs), key informant interviews (KIIs) and focus group discussions (FGD) with participants. IDIs of the participating adolescents will be repeated at endline to examine change over time.

\section{Client and public involvement}

Community members from Phase 1 TPI communities were actively involved in designing TPI during the formative research phase. $^{7}$ This included participating in a learning and design workshop that included discussions on programme content, structure and duration, as well as secondary (or learning) outcomes. In the TPP+ models, 



Figure 3 Randomly selected and assigned wards to treatment and control arms in study districts, CARE Tipping Point Nepal cluster randomised controlled trial.

CARE is implementing, adolescents, their parents and community members are engaged in group dialogues, from which ideas for community events are generated. Adolescent girls are identified to serve as activists in their communities. Community members in the study sites also were involved in piloting all study forms. Dissemination of the findings will include events in the study districts and with Nepali officials.

\section{Sample design}

Sampling and randomisation of primary sampling units

In Nepal, wards (the lowest governmental administrative unit) were the primary sampling units. Using the size of the resident population from the 2011 Census of Nepal, 27 wards were randomly selected with probability proportionate to size from each of two study districts. Each selected ward within each district was randomly assigned to one of the three study arms (Also allowing for balance in the sample across study districts, for programme implementation purposes.), for 18 wards per study arm (figure 3).

\section{Cluster selection and pretrial household census}

Enumerators for the households census mapped selected wards in the field to ensure an accurate household count or to update the count based on information from ward authorities. ${ }^{10}$ Enumerators divided large wards ( $>200$ households) into segments of $\sim 200$ households and selected one segment randomly. The selection of segments within wards also minimised the extent of physical adjacency of programme and control segments and any spill-over of programme effects into control areas.

In selected clusters (wards or ward segments), enumerators conducted a household census. Census forms were administered to the most knowledgeable woman member, or alternatively to any knowledgeable adult member (online supplemental file 1). Enumerators collected contact information and data on the household's caste, religion and language(s) spoken. Marital status, age at marriage and years married were recorded for members above age 8 years. These data generated a sampling frame from which eligible participants for groups in clusters randomly assigned to TPP or TPP+ were identified.

\section{Qualitative cluster selection}

For the qualitative research, the team chose 8 of the 36 randomly selected clusters assigned to TPP or TPP+ in each district. The Nepal study team selected clusters to ensure diversity with respect to treatment (TPP and TPP+ clusters were equally represented) and with respect to ethnicity, religion and language. Given the lack of wardlevel data at the time of cluster selection, CARE Nepal staff shared insights to identify sites that were diverse and thought to have high levels of child marriage. Characteristics of these sites, based on field reports, are shown in table 1 .

\section{Eligibility}

In treatment and control arms, eligible girls and boys were unmarried, 12-16 years, living in selected clusters, with no plans to migrate in the subsequent 24 months. In clusters randomised to receive TPP or TPP+, participation was voluntary for ethical reasons, and so eligible girls and boys in these clusters also were consenting $\mathrm{TPP} / \mathrm{TPP}+$ participants. To measure community norms, eligible adults in treatment and control arms were men and women 25 years or older, living in selected clusters, and with no known plans to migrate in the subsequent 24 months.

For the qualitative research, eligible adolescents were unmarried girls and boys 12-16 years who were living in selected study clusters. Eligible mothers and fathers were male and female parental figures of an adolescent boy or girl who consented to participate in the programme and associated measurement. Eligible community stakeholders were male or female community leaders identified by sponsor organisational contacts in selected clusters. 
Table 1 Characteristics of the qualitative study sites, by study districts in Nepal

\section{Kapilvastu}

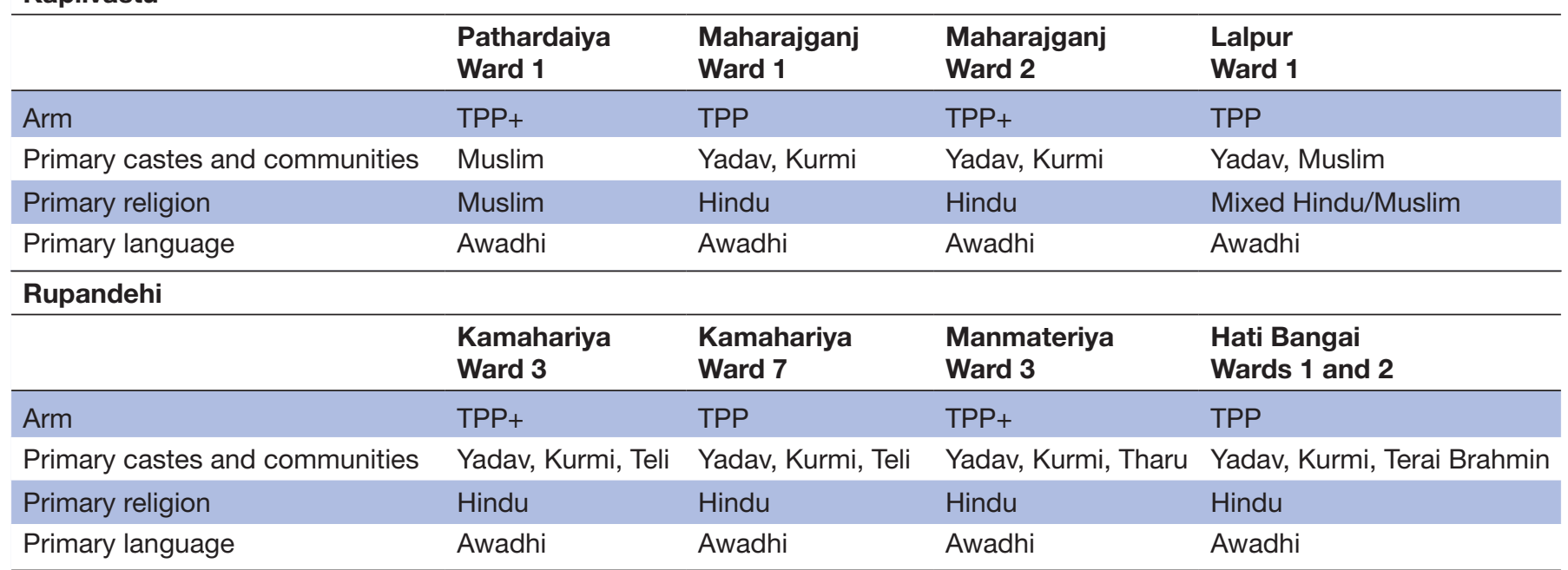

TPP, Tipping Point Programme; TPP+, Tipping Point Program with emphasized social norms change.

\section{Survey sample size and power}

For the survey, each cluster was intended to have 20 adolescent girls and 20 adolescent boys at endline. Assuming a $50 \%$ prevalence of the primary (CEFM) and secondary (agency) outcomes to allow for maximum sample size, a 5\% significance level, $80 \%$ power, an intra-cluster correlation of 0.05 (based on prior work in Bangladesh, ${ }^{11}$ and a $15 \%$ effect size between study arms, 18 clusters per study arm and 54 clusters total were needed. Assuming a greater than $90 \%$ participation rate at baseline and an $85 \%$ retention rate at endline for girls and boys, the target baseline enrolment was 23 girls and 23 boys per cluster, or 1242 girls and 1242 boys in total. For the adult community sample, to assess socialnorms change, assuming $50 \%$ prevalence of CEFMrelated norms and a $15 \%$ change after the intervention, $5 \%$ significance level, $80 \%$ power and $5 \%$ non-response rate, 90 adult women and 90 adult men 25 years or older were needed per arm, for a total sample size of 540 adult women and men.

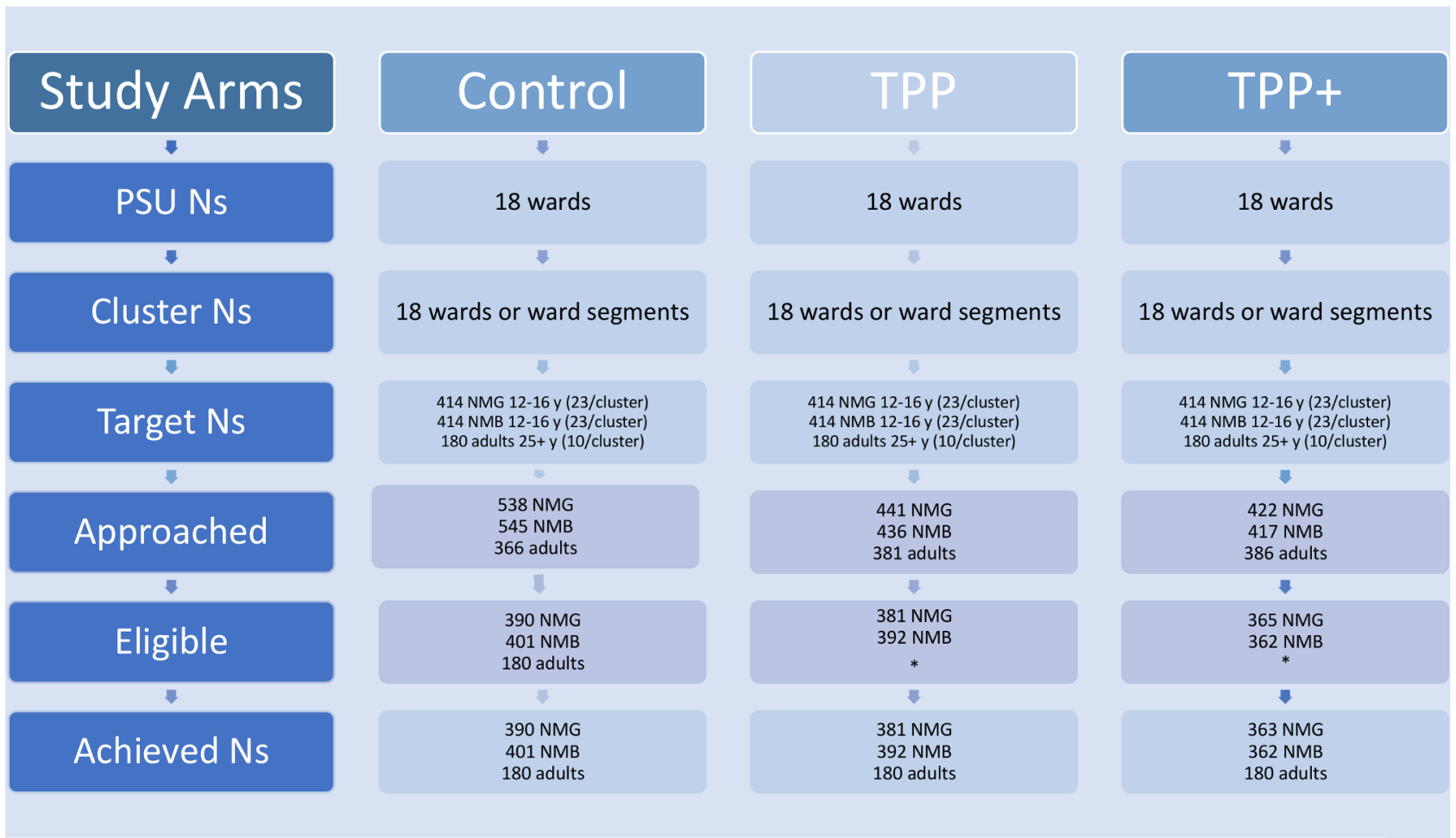

Figure 4 Quantitative sample design and achieved samples at baseline, CARE Tipping Point Impact Evaluation. TPP, Tipping Point Programme; PSU, primary sampling units; NMG, Never-married girl; NMB, Never-married boy. 


\section{Baseline recruitment}

From the census data, names and contact information for eligible, randomly selected adolescent girls $(\mathrm{N}=27)$ and boys $(\mathrm{N}=27)$ in TPP and TPP+ clusters were provided to CARE to form programme groups. Following these randomly ordered lists, CARE Tipping Point project staff recruited and enrolled each eligible adolescent to participate in programming. The local research partner followed up with consenting participants to administer the informed consent and survey until the target number of participants was reached per cluster (23 girls, 23 boys) or all 27 individuals had been approached, whichever came first. Thus, the survey sample represents those who agreed to participate in TPI, and not the population. In control clusters, and for the adult community samples in all clusters, the research team randomly selected eligible participants from cluster-specific lists, invited them to participate in the study, administered the informed consent, and administered the survey to consenting individuals.

For the qualitative research, lists of eligible adolescent girls and boys and their adult care providers were generated from the census data from the eight selected clusters. Lists indicated who had been selected for programme participation to achieve balance in group discussions (see below). Grandparents replaced parents in group discussions when parents had migrated for work.

\section{Achieved samples}

Achieved baseline survey sample sizes were 270 adult women, 270 adult men, 1134 unmarried adolescent girls and 1154 unmarried adolescent boys. The total achieved sample sizes across study arms are summarised in figure 4 . Sampling for the qualitative research continued until the planned number of IDIs, KIIs and FGDs were completed.

\section{Study forms}

Baseline quantitative assessments

The baseline survey in Nepal included 20 modules that measured personal assets/intrinsic agency, instrumental agency, collective agency, social networks and norms, and discrimination and violence as barriers to change (table 2). ${ }^{10}$ Nineteen modules were administered to girls; 16 to boys, and 5 to adult community members. Questionnaires were customised for each sample but maintained high comparability (online supplemental file 2).

\section{Endline quantitative assessments}

With minimal modifications to ensure comparability, baseline survey forms will be readministered at endline, after the 53-month TPP and TPP+ programme period and 8-month period without programming (to assess sustained impact). To minimise attrition, the endline survey will occur when community residents who migrate for work are expected to return for harvesting. Assessors

Table 2 Questionnaire modules and samples interviewed, Care Tipping Point Impact Evaluation in Nepal

\begin{tabular}{|c|c|c|c|c|}
\hline \multirow[b]{2}{*}{ Questionnaire module (source) } & \multirow[b]{2}{*}{ Construct measured } & \multicolumn{3}{|c|}{ Samples receiving } \\
\hline & & Girls & Boys & Adults \\
\hline 1. Self-efficacy & Intrinsic agency & $x$ & $x$ & \\
\hline 2. Aspiration about marriage and education ${ }^{10}$ & & $x$ & $\mathrm{x}$ & \\
\hline 3. Attitudes about gender ${ }^{10}$ & & $x$ & $x$ & $x$ \\
\hline 4. Menstruation knowledge, attitudes, practices & & $x$ & & \\
\hline 5. Knowledge/attitudes about sexual/reproductive health & & $x$ & $\mathrm{x}$ & $x$ \\
\hline 6. Mobility or freedom of movement ${ }^{10}$ & Instrumental agency & $x$ & & \\
\hline 7. Negotiation on education, marriage, mobility & & $x$ & $x$ & \\
\hline 8. Communication and negotiation with parents & & $x$ & $\mathrm{x}$ & \\
\hline 9. Participation in financial activities & & $x$ & & \\
\hline 10. Leadership competence ${ }^{10}$ & & $x$ & $\mathrm{x}$ & \\
\hline 11. Group membership ${ }^{10}$ & Collective agency & $\mathrm{x}$ & $x$ & \\
\hline 12. Cohesion, solidarity and mobilisation skills & & $x$ & $x$ & \\
\hline 13. Participation in events & & $x$ & $x$ & \\
\hline 14. Connectedness & Social networks and norms & $x$ & $\mathrm{x}$ & \\
\hline 15. Social networks ${ }^{10}$ & & $x$ & & \\
\hline 16. Social norms & & $\mathrm{X}$ & $x$ & $x$ \\
\hline 17. Differential treatment of sister & Discrimination and violence as & $x$ & $x$ & \\
\hline 18. Sexual abuse & & $x$ & $x$ & $x$ \\
\hline 19. Peer violence ${ }^{10}$ & & $x$ & $x$ & $x$ \\
\hline 20. Violence, alcohol and drugs & & & $\mathrm{x}$ & \\
\hline
\end{tabular}

NMB, Never-married boys; NMG, Never-married girls; PSU, Primary Sampling Unit. 
Table 3 Qualitative methods and samples interviewed, Care Tipping Point Impact Evaluation in Nepal

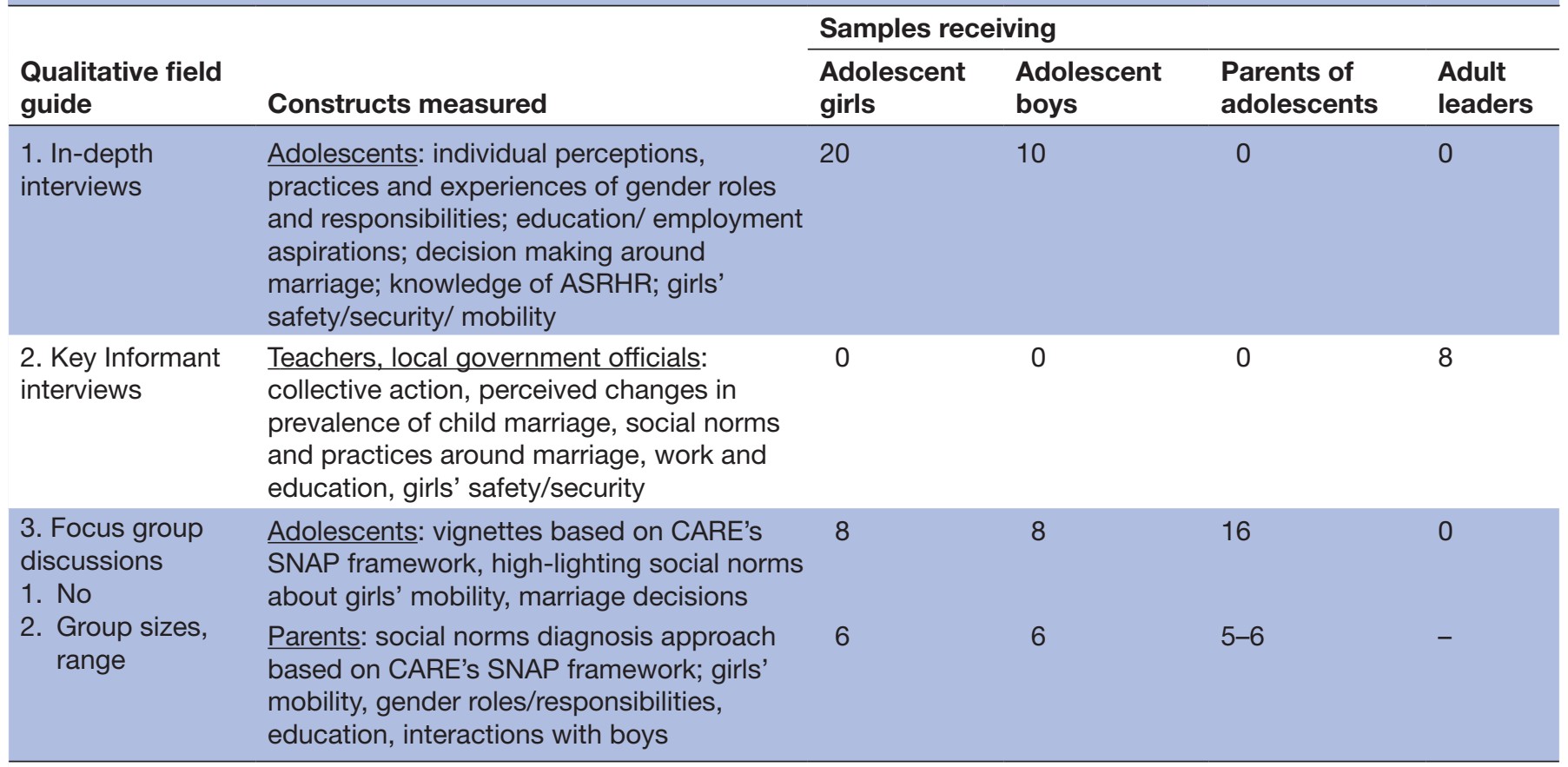

SNAP, social norms analysis plot.

will be blinded to the treatment to which respondents have been exposed. Also, adolescents in programme and control areas will be asked about their exposure to TPP and TPP+ sessions and activities to glean a general understanding of programme fidelity in terms of sessions held as well as potential spill-over of programmatic activities in control clusters.

\section{Baseline qualitative guides}

Baseline qualitative methods included IDIs with 20 adolescent girls and 10 adolescent boys, 8 KIIs with adult community leaders, 16 FGDs with 8 groups of adolescent girls and 8 groups of adolescent boys, and 16 FGDs with parents of adolescents in study communities (table 3, online supplemental file 3). IDIs with adolescents provided narrative data on attitudes and perceptions towards gender roles, life aspirations, girls' safety and security, and girls' mobility. FGDs with adolescents and parents were based on CARE's social norms analysis plot framework, ${ }^{12}$ highlighting social norms surrounding girls' mobility, decision making around marriage, and interaction with boys. KIIs with teachers and local officials gathered data on collective action, perceived changes in the prevalence of child marriage, social norms and practices around marriage, work and education, and girls' safety and security.

\section{Programme monitoring forms}

CARE developed standards for assessing internal implementation fidelity, including for the structure and daily functioning of the programme, to ensure adherence to the intended programme packages and highquality implementation across programme arms (TPP and TPP+). CARE has assessed implementation fidelity through observations of at least $10 \%$ of the sessions every month and feedback from all Tipping Point participants at least every 6 months. These observations and feedback sessions are discussed internally monthly and quarterly to assess the project's fulfilment of these standards. The research partners also are conducting independent monitoring visits, including observations of the quality of the sessions and skill of the facilitators, adherence to the project's implementation manuals, and participants' and communities' response to TP activities and content. The Emory/IDA team are administering monitoring forms in TPP and TPP+ sites twice, at 6 monthly intervals from baseline during programme implementation (online supplemental file 4). Table 4 summarises the forms, samples and topics covered, including questions to understand the potential impacts of COVID-19 risk mitigation strategies on families in TPP and TPP+ study arms. The 24 session-observations elicit information about the fidelity of facilitators to programme content and delivery. Recommendations provided by the research partners are reviewed in quarterly meetings with field facilitators and higher management of the implementing partners.

\section{Training}

For the household census, enumerators received a 2-day training (29-30 April 2019) on the study design and aims, use of mobile data collection equipment, and research ethics. The census occurred 1-29 May 2019, visiting 9289 households and documenting 46578 household members. 
Table 4 Forms for 6 monthly programme monitoring visits, Care Tipping Point Initiative impact evaluation in Nepal

\begin{tabular}{|c|c|c|c|c|c|c|}
\hline Form & Topics & Visits & No per visit & Total & Samples & Sites \\
\hline $\begin{array}{l}\text { Session } \\
\text { observation }\end{array}$ & $\begin{array}{l}\text { Facilitator performance including skill, } \\
\text { fidelity to manual, language }\end{array}$ & 1,3 & 8 & 24 & $\begin{array}{l}\text { Mothers, fathers, } \\
\text { adolescent girls, } \\
\text { adolescent boys }\end{array}$ & $\begin{array}{l}\text { Bagaha, } \\
\text { Pathkauli, } \\
\text { Kamahariya, } \\
\text { Khurhuriya }\end{array}$ \\
\hline $\begin{array}{l}\text { Focused group } \\
\text { discussion } \\
\text { with parents }\end{array}$ & $\begin{array}{l}\text { Economic and social impact of COVID } \\
\text { triggered lockdown on parent-child } \\
\text { communication, gender roles and } \\
\text { expectations and social norms }\end{array}$ & 2 & 2 & 2 & Mothers, Fathers & TBD \\
\hline
\end{tabular}

\begin{tabular}{|c|c|c|c|c|c|c|}
\hline $\begin{array}{l}\text { Event } \\
\text { observation }\end{array}$ & $\begin{array}{l}\text { Participant roles and engagement; } \\
\text { community roles and engagement; } \\
\text { challenges and successes, impact on } \\
\text { girls' collective action due to lockdowns } \\
\text { triggered by COVID }\end{array}$ & 2,3 & 1 & 2 & - & TBD \\
\hline $\begin{array}{l}\text { Focus group } \\
\text { discussion }\end{array}$ & $\begin{array}{l}\text { Group processes and procedures; group } \\
\text { cohesion and solidarity; challenges } \\
\text { and successes; leadership; community } \\
\text { engagement; effects of COVID-19 risk- } \\
\text { mitigation strategies in study clusters }\end{array}$ & 2,3 & 2 & 4 & Adolescent girls & TBD \\
\hline Rolling profiles & $\begin{array}{l}\text { Communication; ASRH; eve teasing } \\
\text { and violence; aspirations; gender roles } \\
\text { and norms; effects of COVID-19 risk } \\
\text { mitigation strategies in study clusters }\end{array}$ & $1,2,3$ & $\begin{array}{l}12 \text { (3 families } \\
\times 4 \text { members) }\end{array}$ & 36 & $\begin{array}{l}\text { Mother, father, } \\
\text { adolescent girl, } \\
\text { adolescent boy }\end{array}$ & $\begin{array}{l}\text { Bagauli, } \\
\text { Jayanagar, } \\
\text { Khurhuriya }\end{array}$ \\
\hline
\end{tabular}

ASRH, Adolescent Sexual and Reproductive Health; TBD, to be determined.

A 10-day training (30 May 2019-8 June 2019) for baseline included training and practice with study forms, tablet-based data collection and data quality. All data collectors received training on research ethics, including CARE's reporting protocols surrounding gender-based violence. The training also included field test of the baseline survey forms in one of the two study districts. In addition to the general training, qualitative data collectors received a 2-day training on gender, rights, empowerment, CEFM and qualitative research methods.

\section{Recruitment and retention}

To recruit and retain participants in the programme and trial, CARE first adapted and tested an intervention package that would engage participants, especially boys and girls. Second, each group agreed on timing and location of meetings with participants to facilitate attendance. Third, a follow-up is triggered for participants who miss two consecutive sessions, for whom Tipping Point facilitators make a home visit to discuss barriers to attendance and to problem-solve re-engagement. Finally, the team plans to implement a two-phase tracing protocol at endline, following guidance on origin-based sampling and phone-tracing techniques from Nepal. ${ }^{13}$ First, during the endline survey, we will conduct brief, in-person proxy interviews with primary caretakers or household members closest to participants who are lost to in-person follow-up at endline. Second, we will conduct brief, postendline interviews over the phone with those same participants using a shortened questionnaire adapted for phone interviews. The goals of this protocol will be to maximise overall retention of programme participants and to minimise any differential retention across study arms that may arise from differential contact with participants in the programme and control arms.

\section{Fieldwork}

All data collectors spoke Nepali and the local languagesAwadhi and Bhojpuri-and were gender-matched with participants. Qualitative data collection occurred 7-20 June 2019. Survey data collection occurred 10 June 2019-10 July 2019.

\section{Data management, access and sharing}

The REDCap mobile app and android tablets permit the secure collection, transfer, and storage of all quantitative study data. Study data are available to trained Emory study staff for cleaning and analysis. Deidentified study data will be stored and shared with collaborating institutions via Emory Box, a password protected, HIPAAcompliant cloud drive. Final study data will be made public by mutual agreement in keeping with CARE and Emory policies regarding data sharing (see ClinicalTrials. gov registry).

\section{Quantitative data analysis}

Primary and secondary outcomes

Table 5 summarises the primary and secondary outcomes and expected impacts of TPP and TPP+ on each outcome.

The primary outcome is CEFM during the programme or freeze period, and if so, the age of first marriage 
Table 5 Expected primary and secondary (intermediate) outcomes of the Tipping Point Impact evaluation in Nepal

\begin{tabular}{|c|c|c|c|c|}
\hline & & Control & TPP & TPP+ \\
\hline CEFM & $\begin{array}{l}\text { Hazard of marriage from baseline to endline } \\
\text { Hazard of gauna from baseline to endline }\end{array}$ & - & $\downarrow$ & $\downarrow \downarrow$ \\
\hline \multicolumn{5}{|l|}{ Secondary outcomes } \\
\hline $\begin{array}{l}\text { Personal assets and } \\
\text { Intrinsic agency }\end{array}$ & $\begin{array}{l}\text { Knowledge of sexual and reproductive health and rights (nine } \\
\text { items, score 0-27) } \\
\text { Aspirations about marriage/education/work (frequencies) } \\
\text { Self-efficacy (11 items, score 0-22; } 13 \text { items for girls, score } \\
\text { 0-28) } \\
\text { Attitudes about gender/gender discrimination/menstruation/ } \\
\text { masculinity (nine items, score 0-27) }\end{array}$ & - & $\uparrow$ & $\uparrow \uparrow$ \\
\hline Instrumental agency & $\begin{array}{l}\text { Mobility of girls (nine items, score 0-18) } \\
\text { Communication with parents (six items, score 0-18) } \\
\text { Leadership competence (nine items, score 0-27) } \\
\text { Participation in financial activities (frequencies) } \\
\text { Participation in decision-making (frequencies) }\end{array}$ & - & $\uparrow$ & $\uparrow \uparrow$ \\
\hline $\begin{array}{l}\text { Girl-centred movement } \\
\text { building and collective } \\
\text { agency }\end{array}$ & $\begin{array}{l}\text { Collective efficacy (nine items, score } 0-15 \text { ) } \\
\text { Collective action }\end{array}$ & - & $\uparrow$ & $\uparrow \uparrow$ \\
\hline $\begin{array}{l}\text { Changes in repressive } \\
\text { social norms }\end{array}$ & $\begin{array}{l}\text { Community social norms (11 items for norms about women } \\
\text { and girls, score 0-33; five items for norms about boys, score } \\
0-15 \text { ) }\end{array}$ & - & $\uparrow$ & $\uparrow \uparrow$ \\
\hline $\begin{array}{l}\text { Reductions in violence } \\
\text { and harassment as } \\
\text { barriers to change }\end{array}$ & $\begin{array}{l}\text { Gender discrimination in the family (five items, score 0-15) } \\
\text { Public violence/harassment against girls }\end{array}$ & - & $\uparrow$ & $\uparrow \uparrow$ \\
\hline
\end{tabular}

Control communities are expected to show little change over the study period due to the concentration of child marriage in these conservative communities and the period of observation. The symbol $\uparrow$ refers to an increase, and the symbol $\uparrow \uparrow$ refers to a more substantial increase. The symbol $\downarrow$ refers to a decrease, and the symbol $\downarrow \downarrow$ refers to a more substantial decrease.

CEFM, child, early and forced marriage; TPP, Tipping Point Programme.

and gauna, or age of cohabitation with the spouse and consummation of the marriage.

Secondary outcomes are organised following the theory of change (figure 2). Outcomes are summative scores for measures of personal assets (knowledge about SRH; membership or leadership in groups), intrinsic agency (aspirations about marriage/education/work; selfefficacy; attitudes about gender roles/gender discrimination/menstruation/masculinity), instrumental agency (mobility of girls; communication with parents; leadership competence; participation in financial activities and decision making), collective agency (collective efficacy; collective action), changes in repressive social norms (community social norms), and reductions in violence and discrimination (gender discrimination in the family; public violence/harassment against girls).

\section{Descriptive analyses of pretrial household census data}

The Emory team was responsible for cleaning and analysis of pretrial census data. Census data on individual household members were used to estimate the percentages of men and women members, by 5-year age groups, who were married, with and without gauna, before the ages of 15 and 18. Married individual household members reported their ages at marriage and gauna, and these data were used to estimate median ages at marriage and gauna by district, gender and age group (table 6). These census data also will be used after endline to compare, by study arm, the characteristics of sample participants at each wave with those in the census sample who would have been eligible for participation but were not included. Any differences between the census sample and study participants by arm will be reported, and considered in interpretations of analyses that assess TPP and TPP+ impacts.

\section{Scale construction and reliabilities}

Individual survey questions (items) for adults, adolescent girls, and adolescent boys will be organised into item sets capturing TPI secondary outcomes. Items will be recoded to be anchored at zero. Missing responses will be coded as missing for univariate analyses of items. Pearson pairwise correlations will ensure that items within sets are mutually correlated and summative scales are reasonable reflections of intended secondary outcomes. An item will be considered for deletion if the magnitude of its pairwise correlation with others in the same item set is close to zero and not significant. Scale reliabilities will be estimated for all outcomes using Cronbach's alpha for each item set or 


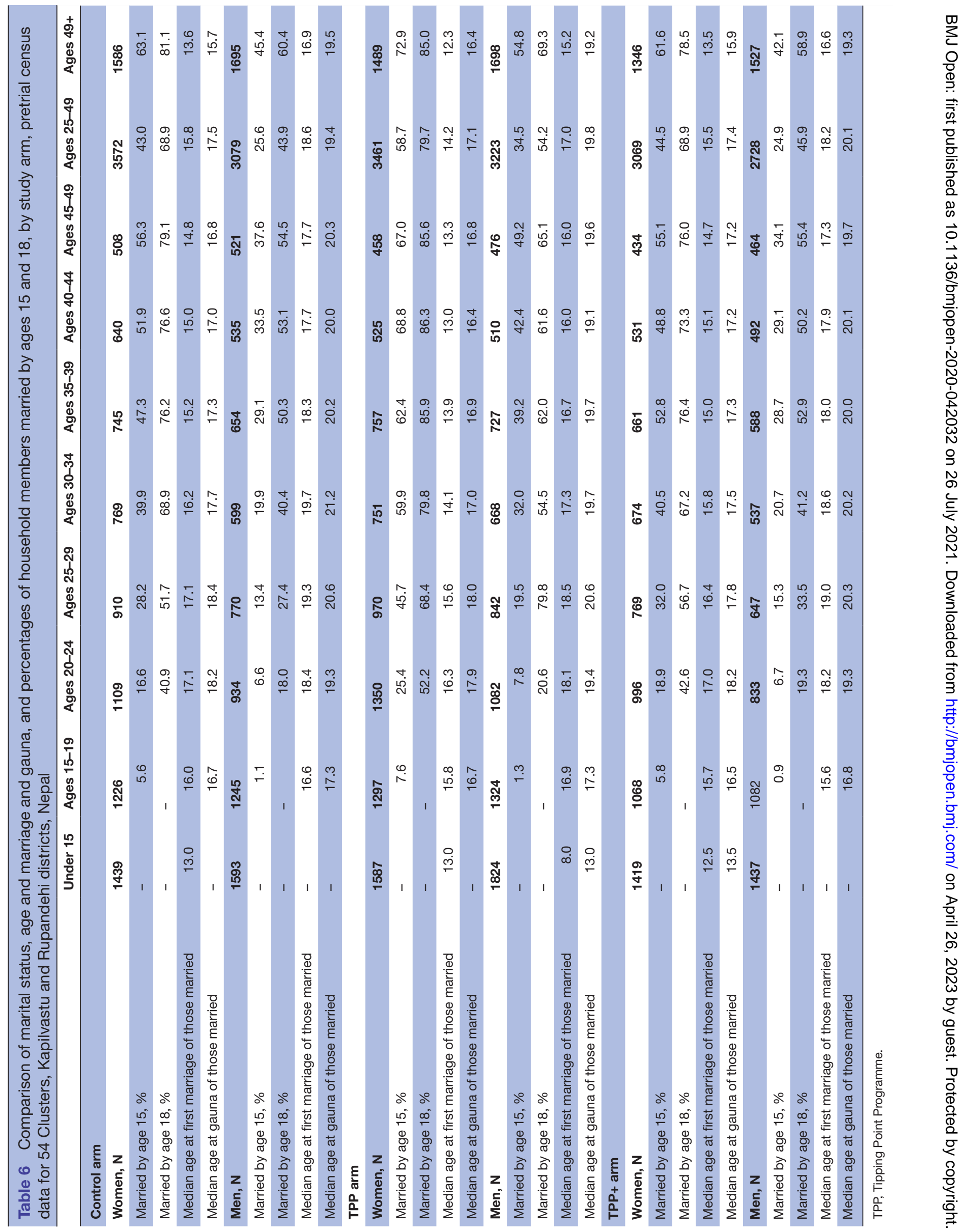


subset after item deletion. For secondary outcomes with a low Cronbach's alpha, we will drop the item with the lowest item-scale reliability. In baseline and endline analyses, we will conduct exploratory and confirmatory factor analysis to determine whether scales for the secondary outcomes are unidimensional or multidimensional.

\section{Baseline descriptive analysis of secondary outcomes and covariates}

Mean scores, and score tertiles for secondary outcomes will be reported. Demographic characteristics and scales for secondary outcomes will be compared across study arms (control, TPP, TPP+) to assess within-sample balance across study arms. Group differences will be assessed using $\chi^{2}$ tests of independence for categorical variables and t-tests for continuous (or quasi-continuous) outcomes, accounting for the cluster-sample design. All descriptive analyses will be completed in Stata V.16.

\section{Time-to-event and intention-to-treat impact assessment}

For the CEFM outcomes, given that the data are right censored, we will estimate marginal Cox proportional odds models ${ }^{1415}$ to estimate the impact of the treatment groups on the hazard of marriage or gauna in months from baseline, relative to the control group, adjusted for covariates. For secondary outcomes, we will estimate (nonparametrically) the average treatment effect (ATE) by computing the difference between their means for intervention participants and non-participants. We will use the difference-in-difference (DiD) regression approach with cluster-robust variance estimators. ${ }^{16}$ We will perform a robustness check of the DiD results using the predicted hazard of marriage or gauna for each secondary outcome as sampling weights. ${ }^{17}$ The DiD model may be extended to assess the impacts of TPP and TPP+, relative to the control group, in a full mediation model, following figure 2.

\section{Addressing observed and unobserved imbalances across study arms}

In the above impact assessments, we address questions about potential non-comparability of the samples across study arms that may be introduced by unintended failure of the randomisation process and/or the voluntary participation of adolescents in TPP and TPP+. First, we will compare characteristics of TPP/TPP+ participants at each wave with those from the census sample in the same study arm who would have been eligible and report any differences. Second, we assess 'balance' on a range of observed characteristics across study arms. If imbalances are observed, we will use propensity score methods ${ }^{18-20}$ that team members have used previously ${ }^{21}$ to generate conditional probabilities of enrolment in TPP and TPP+, given a set of covariates. This method aims to reduce overt biases in the estimated treatment effect due to observed preprogramme differences in participation resulting from the voluntariness of participation for ethical reasons. ${ }^{18}$ We will include covariates in a probit model to predict the likelihood of programme participation, including, for example, district strata, observed variables that are unbalanced across study arms at baseline, and potential differences in COVID-19 risk-mitigation strategies that may be related to the risk of CEFM. From this model, we will estimate propensity scores for participation in TPP and TPP+.

With these estimated propensity scores, we will use the inverse probability of treatment weighting approach to assign weights to treatment and control group members to estimate the ATEs across various outcomes. ${ }^{22}$ To reduce possible residual biases from any misspecifications of the weighted regression models, we will apply covariate adjustment using all covariates except an exogenous instrument, if one can be identified. ${ }^{23}$ To reduce the risk of a type I error associated with testing programme effects on multiple outcomes, we may apply the Bonferroni adjustment by dividing the alpha value by the number of outcomes.

After estimating each of the treatment-effects models, we will visually inspect the extent of overlap in the propensity-score distributions across TPP/TPP+ participantsand non-participants. ${ }^{20}$ The extent of overlap in the propensity score distributions indicates the likelihood that each participant enrolled or not in TPP/TPP+ also has a certain likelihood of enrolment in the other group(s). The greater the overlap of the propensity-score distributions across groups, the greater is the common support and the likelihood of balance ${ }^{19}$ When balance is achieved, preprogramme distributions on the propensity scores and the covariates are likely to be similar, and the dataset provides support for causal inference. ${ }^{19}$

Alongside visual inspection of the propensity-score distributions across groups, we will perform $\chi^{2}$ overidentification tests for balance across groups. Finally, we will perform a test for endogenous treatment effects in each of the analyses. Endogeneity occurs when some unobservable components affect programme enrolment and outcomes of interest, ${ }^{20}$ potentially making inaccurate the estimates of treatment effects. To estimate the ATEs, to inspect overlap, and to assess balance and endogeneity, we will use the modules teffects and eteffects ipwr $a$ in Stata V.16. ${ }^{24}$ Finally, we will interpret findings of treatment effects judiciously considering observed differences across study arms, visual inspection of propensity score distributions, overidentification tests for balance across groups, and test for endogeneous treatment effects.

\section{Dose-response analysis in the TPP and TPP+ groups}

We will use monitoring data collected by CARE Nepal to assess the adjusted associations of programme participants' session attendance with primary and secondary outcomes. We will assess effect modification of sessions attended by treatment arm on a multiplicative scale for the primary outcomes and on an additive scale for the secondary outcomes. To understand the dynamics of programme participation and dropout, we will use logistic regression to model the pretrial determinants of nonparticipation in the TPP or TPP+ models (district, cluster, 
household socioeconomic conditions, employment status of household head, household size, etc).

Sensitivity analysis on the treatment effect in the presence of noncompliance and contamination

We will assess the robustness of the inferences regarding the treatment effects using an instrumental variable approach with the randomisation as the instrumental variable. $^{25}$

\section{Qualitative data analysis}

Baseline qualitative data analysis

Emory's team revised a qualitative codebook developed by icddr,b for Bangladesh ${ }^{26}$ to streamline existing codes and add new codes on emergent themes from the Nepal data (online supplemental file 5). Several rounds of codebook edits were made, first through careful reading of 10 transcripts and discussions with CARE and icddr,b; second, after two rounds of intercoder reliability testing (evaluated using Cohen's kappa ${ }^{27}$ among three Emory team members using seven transcripts, and finally, after coding 20 transcripts across two sites. Team debriefs were used to resolve discrepancies and make minor edits to codes and definitions, after which the same three-member team divided the remaining 50 transcripts to code individually. All coding, cross-classification and inter-coder reliability testing was performed in MAXQDA V.18 (Berlin, Germany) by this team.

A narrative analysis of IDIs with adolescents began with memos of each transcript summarising themes of ASRH, aspirations, marriage, mobility, safety and security. Descriptive analysis of social norms data across all 70 transcripts was performed by crossing each major theme with relevant norms codes (normative expectations, empirical expectations, sanctions/sensitivity to sanctions, exceptions). Thick descriptions were generated for five norms CARE identified through formative research. ${ }^{26}$ Documentation of analytical process through memos and triangulation across samples and data collection methods permitted validation at each analytic phase.

\section{Longitudinal qualitative data analysis}

We will repeat the above process with endline data and conduct structured comparisons between baseline and endline data within themes as well as by gender, site, caste/ community and any other emergent variable found to be relevant to the change process. Finally, we will generate thick descriptions for all codes representing the theory of change and apply causal chain analysis ${ }^{28}$ to interrogate the components of the theory of change.

\section{DISCUSSION}

Limitations and strengths of the TPI impact evaluation design in Nepal

- Despite randomising wards to study arms, samples may differ on selected characteristics (school type, caste, religion, girls' literacy), which we will control for in analyses.

- Field staff reported challenges collecting accurate data on age to determine eligibility, which the team addressed with repeated verification. Data on age were first gathered during the household enumeration exercise by IDA-trained staff and data collectors. This information was then verified, and inconsistencies resolved, while CARE and implementing partner staff were forming groups of eligible programme participants to ensure criteria for intervention inclusion were met.

- As with age, accurate estimates of age at marriage required repeat verification and triangulation during data cleaning and analysis. Triangulation of age and age of marriage during data cleaning and analysis was achieved by including questions in the enumeration form (current age, age at marriage, years married) that allowed for consistency checks in age reporting and by assessing concordance in years married with husband-wife dyads.

- The Nepal TPI study team faced challenges recruiting participants for boys' and girls' groups in the intervention study arms, so the team sought informed consent from and surveyed only those who agreed to participate in TPP/TPP+. This decision may have resulted in potentially non-representative samples of boys' and girls' in intervention arms, differences in observed and unobserved characteristics of adolescents in treatment and control clusters, the inability to construct sampling weights, and the need to generalise findings to the study sample. Despite this caveat, following a random probability sample of adolescents in the control clusters to understand their trajectories in primary and secondary outcomes in the absence of TPP/TPP+ is important. Also, we have proposed robust methods to diagnose differences between programme participants and otherwise eligible household census members who did not participate. We also have proposed methods to address systematic and meaningful imbalances in observed characteristics across treatment and control arms and to test for the influence of unobserved characteristics (endogeneity) in ATEs. We will interpret these findings judiciously in light of the full set of findings.

- Differential attrition across study arms may arise at endline from the intensive follow-up of TPP and TPP+ participants during the 18 months of programming after baseline and the 30 months of non-contact with participants in the control arm after baseline. After endline, when the extent of attrition is known for all samples in all study arms, we will implement a targeted tracing protocol to mitigate differences in attrition.

Despite these limitations, the TPI Impact Evaluation in Nepal has notable strengths.

- The mixed-methods design allows for triangulation of qualitative and quantitative findings. 
- The three-arm C-RCT design permits assessment of the incremental impacts of the emphasised socialnorm change component.

- Robust field strategies and analytical strategies are proposed to mitigate identified risks and caveats in the study design.

\section{Implications of the study for programme implementation and scale-up}

The CARE TPI is a novel, integrated, social norms and girl-led movement-building initiative that is designed to address the causes of CEFM through dialogue with girls, boys and community members. The initiative is being undertaken in communities of Nepal with high levels of child marriage despite moderate standards of living. The impact evaluation integrates rigorous qualitative and quantitative methods in a cluster randomised controlled design, allowing for inferences to be made within a sample that is intended to be broadly similar to the population of the two study districts, suggesting that the trial may offer insights to the population in these areas. If found to be effective in the study area, the TPI will have enormous potential for national scale-up as well as adaptation to other settings where CEFM remains prevalent.

\section{Ethics and dissemination}

CARE Nepal has ongoing approval from the Social Welfare Council of the Nepali government to operate in the study districts. The Emory University Institutional Review Board (IRB00109419) and Nepal Health Research Council (161 2019) approved the study. The team is following UNICEF guidelines for ethical research involving children, ${ }^{29}$ which corroborate standard ethical guidance $^{30}$ and ensure a child-centred focus. ${ }^{31}$ CARE Guidelines for Interviewing Children and gender-based violence communications guidelines also are being followed. ${ }^{32}$ Written consent forms for adults, as well as parental consent and child assent forms for children (online supplemental file 6), were developed following those from RTR-GEP impact evaluation in Nepal. ${ }^{33}$ The data from this study are available from the corresponding author on reasonable request. All other research materials are included in this published article and its online supplemental information files.

\section{Author affiliations}

${ }^{1}$ Global Health \& Sociology, Emory University School of Public Health, Atlanta, Georgia, USA

${ }^{2}$ Global Health, Emory University School of Public Health, Atlanta, Georgia, USA

${ }^{3}$ Psychology, Emory University, Atlanta, Georgia, USA

${ }^{4}$ Gender Justice Team, CARE USA, Atlanta, Georgia, USA

${ }^{5}$ Interdisciplinary Analysts (IDA), Kathmandu, Nepal

${ }^{6}$ International Centre for Diarrhoeal Disease Research Bangladesh, Dhaka, Bangladesh

${ }^{7}$ Health Equity and Rights Team, CARE USA, Atlanta, Georgia, USA

\section{Twitter Cari Jo Clark @cari_jo_clark}

Collaborators Tipping Point Team, CARE: Shikha Sunuwar, Rajan Subedi, Dipendra Raj Sharma, Tirzah Brown; Interdisciplinary Analysts (IDA): Mr. Hiranya Baral, Ms. Suvechha Ghimire, Mr. Digvijay Mishra, Mr. Sandeep Thapa, Mr. Santosh Kumar
Karki, Ms. Akriti Rana, Mr. Pankaj Pokhrel, Ms. Prakriti Adhikary, Mr. Tikaram Basnet, Ms. Nishu Aryal, Mr. Ram Ishwor Yadav, Ms. Mamta Hamal, Ms. Sarala Regmi, Mr. Anil Chaudhary, Ms. Dilmaya Dhakal, Mr. Gajendra Prasad Sah, Ms. Barsha Glan, and Mr. Nischal Raj Dawadi.

Contributors KMY: conceptualisation, data curation, funding acquisition, investigation, methodology, project administration, resources, software, supervision, visualisation, writing-original draft, writing- review and editing. IB: data curation, project administration, writing-original draft, writing-review and editing. YFC: methodology, writing-original draft, writing-review and editing. CC: conceptualisation, data curation, funding acquisition, investigation, methodology, supervision, writing-original draft, writing-review and editing. SK: conceptualisation, funding acquisition, project administration, writing-review and editing, ZK: formal analysis, investigation, writing-original draft, writing-review and editing. AL: supervision, writing-original draft, writing-review and editing. MAM: conceptualisation, methodology, writing-review and editing. RTN: conceptualisation, methodology, writing-review and editing. KP: conceptualisation, methodology, writing-review and editing. AT: conceptualisation, methodology, writing-review and editing. SS: methodology, validation, investigation, resources, data curation, writing-review and editing, supervision. SG: methodology, validation, investigation, resources, data curation, project administration. AS: conceptualisation, funding acquisition, project administration, supervision, writing-review and editing. All authors have read and approved the manuscript.

Funding The Nepal Tipping Point Ttrial is supported through a research grant from the Kendeda Fund to CARE USA and subawarded to Emory University.

Disclaimer The funder played no role in the design of the study; nor in the collection, analysis, or interpretation of the data; nor in the writing of the manuscript.

Map disclaimer The inclusion of any map (including the depiction of any boundaries therein), or of any geographic or locational reference, does not imply the expression of any opinion whatsoever on the part of BMJ concerning the legal status of any country, territory, jurisdiction or area or of its authorities. Any such expression remains solely that of the relevant source and is not endorsed by BMJ. Maps are provided without any warranty of any kind, either express or implied.

Competing interests None declared.

Patient consent for publication Not required.

Provenance and peer review Not commissioned; externally peer reviewed.

Supplemental material This content has been supplied by the author(s). It has not been vetted by BMJ Publishing Group Limited (BMJ) and may not have been peer-reviewed. Any opinions or recommendations discussed are solely those of the author(s) and are not endorsed by BMJ. BMJ disclaims all liability and responsibility arising from any reliance placed on the content. Where the content includes any translated material, BMJ does not warrant the accuracy and reliability of the translations (including but not limited to local regulations, clinical guidelines, terminology, drug names and drug dosages), and is not responsible for any error and/or omissions arising from translation and adaptation or otherwise.

Open access This is an open access article distributed in accordance with the Creative Commons Attribution Non Commercial (CC BY-NC 4.0) license, which permits others to distribute, remix, adapt, build upon this work non-commercially, and license their derivative works on different terms, provided the original work is properly cited, appropriate credit is given, any changes made indicated, and the use is non-commercial. See: http://creativecommons.org/licenses/by-nc/4.0/.

ORCID iD

Kathryn M Yount http://orcid.org/0000-0003-1917-1574

\section{REFERENCES}

1 Raj A, McDougal L, Rusch MLA. Changes in prevalence of girl child marriage in South Asia. JAMA 2012;307:2027-9.

2 Raj A, Boehmer U. Girl child marriage and its association with national rates of HIV, maternal health, and infant mortality across 97 countries. Violence Against Women 2013;19:536-51.

3 Godha D, Hotchkiss DR, Gage AJ. Association between child marriage and reproductive health outcomes and service utilization: a multi-country study from South Asia. J Adolesc Health 2013;52:552-8.

4 Yount KM, Crandall A, Cheong YF. Women's age at first marriage and long-term economic empowerment in Egypt. World Dev 2018;102:124-34. 
5 Raj A. When the mother is a child: the impact of child marriage on the health and human rights of girls. Arch Dis Child 2010;95:931-5.

6 Pandey S. Persistent nature of child marriage among women even when it is illegal: the case of Nepal. Child Youth Serv Rev 2017;73:242-7.

7 Karim N, Greene M, Picard M. The cultural context of child marriage in Nepal and Bangladesh: findings from cares tipping point project. Community participatory analysis. Reserch report, 2016.

8 Yount KM, Krause KH, Miedema SS. Preventing gender-based violence victimization in adolescent girls in lower-income countries: systematic review of reviews. Soc Sci Med 2017;192:1-13.

9 Chae S, Ngo T. The global state of evidence on interventions to prevent child marriage. GIRL center research brief no 1. New York: Population Council, 2017.

10 Khan Z, Jackson E, Clark CJ. Supporting girls' education: an evaluation of 'Room to Read' in Nepal Emerging baseline findings Executive Summary. London: GAGE/ODI, 2018.

11 Parvin K, Nunna T, Mamun M. Tipping point project: report of the baseline study findings from Bangladesh: icddr, $b$ and CARE USA, 2020.

12 CARE USA. Applying Theory to Practice: CARE's Journey Piloting Social Norms Measures for Gender Programming: Cooperative for Assistance and Relief Everywhere, Inc, 2017.

13 Ghimire DJ, Williams NE, Thornton A, et al. Strategies for originbased surveying of international migrants. J Ethn Migr Stud 2019:45:1185-206.

14 Gail MH, Wieand S, Piantadosi S. Biased estimates of treatment effect in randomized experiments with nonlinear regressions and omitted covariates. Biometrika 1984;71:431-44.

15 Lin DY, Wei LJ. The robust inference for the COX proportional hazards model. J Am Stat Assoc 1989;84:1074-8.

16 Liang K-YEE, Zeger SL. Longitudinal data analysis using generalized linear models. Biometrika 1986;73:13-22.

17 Branas CC, Cheney RA, MacDonald JM, et al. A difference-indifferences analysis of health, safety, and greening vacant urban space. Am J Epidemiol 2011;174:1296-306.

18 Rosenbaum PR, Rubin DB. The central role of the propensity score in observational studies for causal effects. Biometrika 1983;70:41-55.
19 Shadish WR, Steiner PM. A primer on propensity score analysis. Newborn Infant Nurs Reviews 2010;10:19-26.

20 StataCorp. Stata Treatment-Effects reference manual: potential Outcomes/Counterfactual outcomes Stata: release 16. statistical software. College Station, TX: StataCorp LLC, 2019.

21 Yount KM, Cheong YF, Khan Z, et al. Women's participation in microfinance: effects on women's agency, exposure to partner violence, and mental health. Soc Sci Med 2021;270:113686.

22 Hirano K, Imbens GW, Ridder G. Efficient estimation of average treatment effects using the estimated propensity score. Econometrica 2003;71:1161-89.

23 DE H, Imai K, King G. Matching as nonparametric preprocessing for reducing model dependence in parametric causal inference. Political Analysis 2007;15:199-236.

24 StataCorp. Stata statisical software: release 16. College Station, TX: StatCorp LP, 2019

25 Tchetgen Tchetgen EJ, Walter S, Vansteelandt S, et al. Instrumental variable estimation in a survival context. Epidemiology 2015;26:402-10.

26 CARE USA. The cultural context of Child Marriage in Nepal and Bangladesh- Findings from CARE's Tipping Point Project community Participatory Analysis: Cooperative for Assistance and Relief Everywhere, Inc; 2016.

27 Cohen J. A coefficient of agreement for nominal scales. Educ Psychol Meas 1960;20:37-46.

28 Miles MB, Huberman AM, Saldana J. Qualitative data analysis: a methods Sourcebook. 3rd edn. SAGE Publications, 2014.

29 Graham A, Powell MA, Anderson D. Ethical research involving children: UNICEF office of Research-Innocenti Florence 2013.

30 Bell N. Ethics in child research: rights, reason and responsibilities. Child Geogr 2008;6:7-20.

31 Graham A, Powell M, Taylor N. Ethical research involving children. Florence, Italy: UNICEF Office of Research - Innocenti, 2013.

32 Widman L, Choukas-Bradley S, Helms SW, et al. Sexual communication between early adolescents and their dating partners, parents, and best friends. J Sex Res 2014;51:731-41.

33 Bergenfeld I, Clark CJ, Kalra S. Tipping point program impact evaluation: baseline study findings in Nepal. Atlanta, GA: Care USA and Emory University, 2019. 